\title{
Spiral turbulence and spatiotemporal chaos: characterization and control in two excitable media
}

\author{
Rahul Pandit ${ }^{\mathrm{a}, \mathrm{b}, *}$, Ashwin Pande $\mathrm{a}^{\mathrm{a}, 1}$, Sitabhra Sinhaa ${ }^{\mathrm{a}, \mathrm{b}, 2}$, \\ Avishek Sen ${ }^{\mathrm{c}, 3}$ \\ ${ }^{a}$ Center for Condensed Matter Theory, Department of Physics, Indian Institute of Science, \\ Bangalore 560 012, India \\ ${ }^{\mathrm{b}}$ Condensed Matter Theory Unit, Jawaharlal Nehru Center for Advanced Scientific Research, \\ Bangalore 560 064, India \\ ${ }^{\mathrm{c}}$ Department of Physics, St. Stephens College, University of Delhi, Delhi 110 007, India
}

\begin{abstract}
We give an overview of our studies of spiral turbulence and spatiotemporal chaos in partialdifferential-equation models for two excitable media: (a) the oxidation of carbon monoxide on

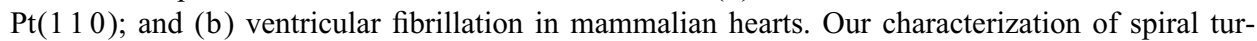
bulence and spatiotemporal chaos in these models leads us to an efficient scheme for controlling such chaos. We discuss this scheme and its application for electrical defibrillation.
\end{abstract}

Keywords: Spiral turbulence; Spatiotemporal chaos; Excitable media

\section{Introduction}

Spiral waves occur in a variety of excitable media. They are found, e.g., in the oxidation of $\mathrm{CO}$ on $\mathrm{Pt}\left(\begin{array}{lll}1 & 1\end{array}\right)$ [1] and are believed to be the principal cause of ventricular

\footnotetext{
* Corresponding author. Center for Condensed Matter Theory, Department of Physics, Indian Institute of Science, Bangalore 560 012, India. Fax: +91-80-334-1683.

E-mail address: rahul@physics.iisc.ernet.in (R. Pandit).

${ }^{1}$ Present address: Department of Mathematics, University of Maryland, College Park, Maryland, USA.

${ }^{2}$ Also at Poorna Prajna Institute of Scientific Research, Bangalore, India.

${ }^{3}$ Present address: Indian Institute of Management, Ahmedabad, India.
} 
fibrillation (VF) [2], a type of cardiac arrhythmia which is responsible for one out of every six deaths in the USA. As we discuss below, the mathematical models [3-5] for these two phenomena are similar. We characterize the spatiotemporal chaos here and the nonequilibrium statistical steady states associated with the breakup of spiral waves. We show how to control this spiral turbulence, which is especially important for ventricular fibrillation. Our control method suggests a defibrillation scheme which uses low-amplitude voltage pulses [5].

When a mixture of $\mathrm{CO}$ and $\mathrm{O}$, both at extremely low partial pressures, is heated to $\simeq 400 \mathrm{~K}$ in a chamber containing a single-crystal wafer of platinum [surface $\operatorname{Pt}\left(\begin{array}{lll}1 & 1 & 0\end{array}\right)$ ], these gases adsorb onto the crystal surface and react to form $\mathrm{CO}_{2}$. The concentration patterns of the adsorbed gases, imaged by photo electron emission microscopy (PEEM), show target patterns, traveling pulses, states with steadily rotating spiral waves, and turbulent states with spiral creation and annihilation [1]. In this temperature regime, the system displays excitability, i.e., there is a threshold concentration of $\mathrm{O}$ below which the $\mathrm{CO}$-covered $\mathrm{Pt}$ surface is stable against subthreshold increases in the local $\mathrm{O}$ concentration. Superthreshold increases trigger a large transient increase in the local $\mathrm{O}$ concentration and may lead to traveling waves and rotating spirals of adsorbed $\mathrm{O}$.

The mammalian heart is an electromechanical organ which pumps blood via rhythmic contractions of the atria and ventricles. The heart wall is an excitable medium that supports the passage of regular contraction waves across it. Cardiac cells become depolarized when stimulated beyond a certain threshold potential. During the course of excitation, the cells remain refractory to new stimuli. Upon recovering their resting potential the cells are ready to be excited again. Each cardiac cell is electrically coupled to its neighbors; hence excitation in one region initiates a wave that propagates through the entire heart. Irregularities in the spatiotemporal behavior of this system are manifested as cardiac arrhythmias. Ventricular fibrillation, the most dangerous of all arrhythmias, results from the emergence of a spiral wave which can spontaneously degenerate into multiple spirals, a phenomenon termed spiral breakup. Repeated spiral breakup completely obscures the regular pulses from the sinus node, the natural pacemaker of the heart. Death occurs in a few minutes unless defibrillation is done immediately. This usually consists of massive electrical shocks applied either to the chest or to the heart surface in order to depolarize the entire heart, thereby bringing it to a quiescent state from which the sinus node can again take over. Electrical defibrillation may sometimes damage the heart and occasionally induce another episode of VF. Thus, methods which can defibrillate with much less damage are of great interest.

The remaining part of this paper is organized as follows: Section 2 describes the partial differential equations that are used to model the oxidation of $\mathrm{CO}$ on $\mathrm{Pt}\left(\begin{array}{lll}1 & 10\end{array}\right)$ and ventricular fibrillation; the similarity of these equations brings out the connection between these widely different problems. Section 3 gives an overview of our principal results and conclusions. 


\section{Models and calculations}

The essential connection between the two physical problems we consider here is that they are described by reaction-diffusion partial differential equations (PDEs). The parameters in these equations are such that the systems are excitable, i.e., subthreshold perturbations of quiescent states decay; however, superthreshold perturbations lead to a large, transient excursion which lasts for some time during which the system is refractory, i.e., it does not respond to further perturbations. In the following two subsections, we discuss the PDEs used to model (a) the oxidation of CO on $\mathrm{Pt}\left(\begin{array}{lll}1 & 1 & 0\end{array}\right)$ and (b) ventricular fibrillation and show how a study of the former can be used fruitfully to develop an understanding of the latter.

We use the model of Ref. [6] for the oxidation of CO on $\operatorname{Pt}\left(\begin{array}{lll}1 & 1 & 0\end{array}\right)$ which consists of the following PDEs in two spatial dimensions $\mathbf{x}$ :

$$
\frac{\partial u}{\partial t}=\nabla^{2} u-\frac{1}{\varepsilon} u(u-1)[u-(v+b) / a] ; \quad \frac{\partial v}{\partial t}=F(u)-v ;
$$

here the fields $u$ and $v$ are related to $\mathrm{CO}$ and $\mathrm{O}$ coverages [6], $a, b$ and $\epsilon$ are control parameters related to rate constants, etc., for the chemical reactions involved, $t$ denotes time, and $F(u)=0$ if $u<\frac{1}{3}, F(u)=1-6.75 u(u-1)^{2}$ if $\frac{1}{3} \leqslant u<1$ and $F(u)=1$ if $u \geqslant 1$. The numerical studies of Ref. [6] have yielded a stability diagram for the statistical steady states of Eq. (1) in the $b-\epsilon$ plane with $a=0.84$ : As $\epsilon$ is increased from 0 , say with $b=0.07$, a transition occurs from a state $\mathrm{S}$, with rigidly rotating spirals, to another state $\mathrm{M}$ with meandering spirals; on further increasing $\varepsilon, \mathrm{M}$ evolves into states T1 and T2 that exhibit spiral turbulence [6]. Our study [4] of model (1) focuses on the states S, M, T1, and T2 and the transitions between them (Section 3 ).

Mathematical models for the simulation of cardiac activity [2] are, typically, reactiondiffusion PDEs involving the transmembrane potential and ionic currents, governed by voltage-gated ion channels. The details of the ionic currents used set apart one model from another. We concentrate on the Beeler-Reuter (BR) [7] and Panfilov [8] models. The reaction-diffusion equations used in models for ventricular fibrillation are of the form

$$
\frac{\partial V}{\partial t}+\frac{I_{B R}}{C}=D \nabla^{2} V
$$

where $V$ is the transmembrane potential, $C$ the capacitance density of the heart wall $\left(\simeq 1 \mu \mathrm{F} / \mathrm{cm}^{2}\right), I$ the instantaneous total ionic current, and $D$ the conductivity. The subscript $B R$ denotes the Beeler-Reuter model in which $I_{B R}$ comprises a transient outward potassium current $I_{K}$, a time-activated outward current $I_{x}$, a fast inward sodium current $I_{N a}$, and a slow inward calcium current $I_{s}$ (all currents in $\mu \mathrm{A} / \mathrm{cm}^{2}$ and voltages in $\mathrm{mV}$ ). These currents depend nonlinearly on $V$ and 6 time-dependent gating variables, that describe the opening and closing of the ion channels, given in turn by ordinary differential equations [7]. The rate constants for two of the gating variables involve a parameter $\sigma$. It has been shown [7] that if $\sigma$ is varied from 0.5 to 1 there is an onset of spiral breakup in the BR model; thus we use $\sigma=0.7$.

The Panfilov model [8] is far simpler than the BR model and considers the activation variable $e$, which represents the transmembrane potential of the cells at point $\mathbf{x}$ and 
time $t$, and the recovery variable $g$, which represents the membrane conductance at the same point. The PDEs for $e$ and $g$ are

$$
\partial e / \partial t=\nabla^{2} e-f(e)-g ; \quad \partial g / \partial t=\varepsilon(e, g)(k e-g) .
$$

The function $f(e)$, which specifies fast processes (e.g., the initiation of the action potential), is piecewise linear: $f(e)=C_{1} e$, for $e<e_{1}, f(e)=-C_{2} e+a$, for $e_{1} \leqslant e \leqslant e_{2}$, and $f(e)=C_{3}(e-1)$, for $e>e_{2}$; the physically appropriate parameter values are [8] $e_{1}=0.0026, e_{2}=0.837, C_{1}=20, C_{2}=3, C_{3}=15, a=0.06$ and $k=3$; and $\varepsilon(e, g)$, which determines the dynamics of the recovery variable, is $\varepsilon(e, g)=\varepsilon_{1}$ for $e<e_{2}, \varepsilon(e, g)=\varepsilon_{2}$ for $e>e_{2}$, and $\varepsilon(e, g)=\varepsilon_{3}$ for $e<e_{1}$ and $g<g_{1}$ with $g_{1}=1.8, \varepsilon_{1}=1 / 75, \varepsilon_{2}=1.0$, and $\varepsilon_{3}=0.3$.

We solve the PDEs by using the numerical schemes described in Refs. [4,5]. These schemes discretize the PDEs on a square or cubic grid of points in space, use both periodic (PBC) and no-flux boundary conditions (NBC) for model (1) and only NBC for models (2) and (3), and for time integration Cash-Carp or Barkley methods [4] for model (1) and forward-Euler schemes for models (2) and (3). The spatial grid consists of $L \times L$ points in dimension $d=2$ and $L \times L \times L_{z}$ points if $d=3$. Suitable initial conditions must be chosen to obtain states with spirals [4,5]; e.g., a broken plane wave evolves into a state displaying spiral turbulence in the two models for ventricular fibrillation.

\section{Results and conclusions}

Our studies of the oxidation model (1) yield several interesting results: The state $\mathrm{M}$ and the transitions from it to $\mathrm{T} 1$ and $\mathrm{S}$ depend on the initial conditions [1] and the boundary conditions. Our initial conditions yield spirals in the statistical steady state. We study periodic boundary conditions (PBC) and Neumann boundary conditions (NBC). These lead to distinct meandering states MP and MN, respectively. MN consists of one large meandering spiral (Fig. 1(a)), whereas MP consists of large meandering spirals coexisting with a finite concentration of small defects (Figs. 1(b) and 2). The temporal evolution of $\mathrm{MN}$ is quasiperiodic [4] and we find indeed that the largest Lyapunov exponent $\lambda_{m} \simeq 0$ here. By contrast MP is chaotic since $\lambda_{m}>0$ for it, principally because of the disorderly motion of the small defects shown in Fig. 1(b). The MN-T1 transition is first order [4]: e.g., the defect number density jumps discontinuously here at $\epsilon \simeq 0.057$. The MP-T1 transition is continuous and can be characterized by an order parameter which can be extracted from $S(\mathbf{k})=1 / 4 \pi^{2}\left\langle\left|\int d^{2} \mathbf{x} e^{\mathrm{i} k \cdot \mathbf{x}} u(\mathbf{x}, t)\right|^{2}\right\rangle$ (angular brackets denote a time average): In a state with large spirals, $S(\mathbf{k})$ displays a secondary peak at $|\mathbf{k}|=k_{c}$, where $k_{c}^{-1}$ is related to the wavelength of the spiral arm. This peak rises continuously from 0 as the system moves from the state $\mathrm{T} 1$ to the state MP. The MP-S transition is also continuous; e.g., $\lambda_{m}$ goes to zero as a power of $\left(\epsilon-\epsilon_{c}\right)$, where $\epsilon_{c}$ is the critical value at which the state $S$ appears. A detailed analysis of our results is contained in Ref. [4]. Here we just highlight the qualitative difference between MP and MN which arises because small spiral defects are absorbed 

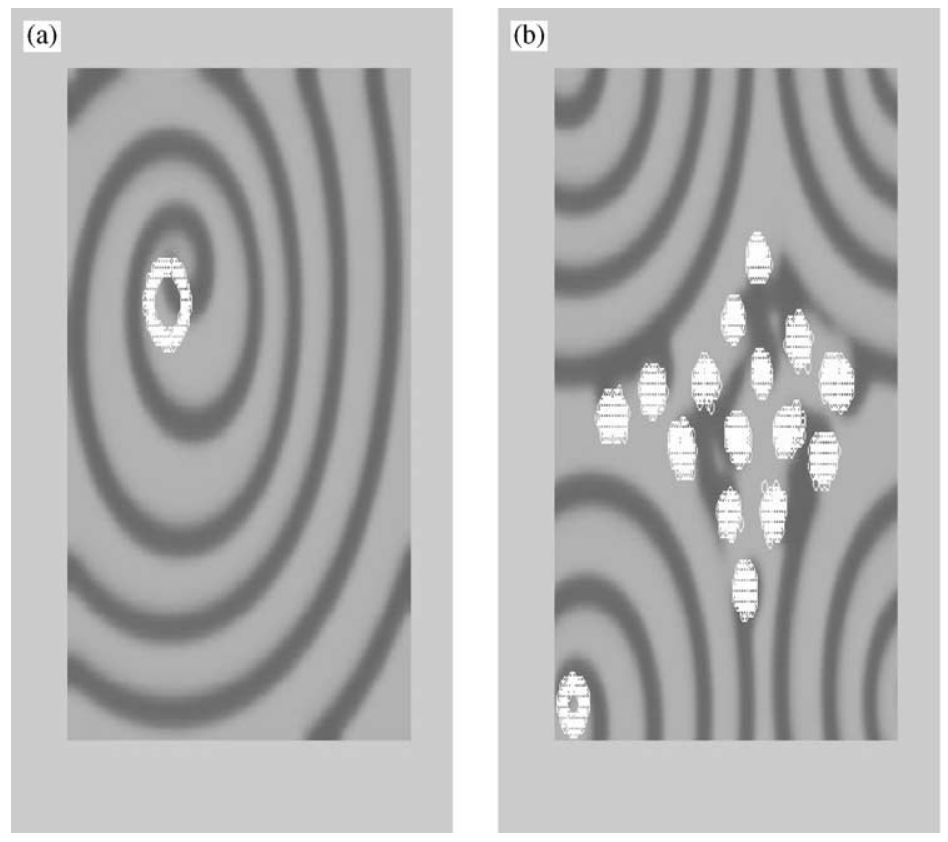

Fig. 1. Gray-scale plots of the $u$-field in (a) the single-spiral state $\mathrm{MN}$ and (b) the inhomogeneous state MP at $\epsilon=\frac{1}{15.9} \simeq 0.0629$. Trajectories of the spiral cores or small-defect cores, superposed in white, indicate phase separation in MP.

by Neumann boundary conditions. We will use this insight in developing defibrillation schemes for models (2) and (3).

We turn now to our results for the ventricular-fibrillation models (2) and (3). We begin with the Panfilov model (3). The onset of spatiotemporal chaos and spiral breakup is illustrated in Fig. 3. As $\varepsilon_{1}$ decreases, the pitch of the spirals decreases till states with broken spirals obtain. For example, if $\varepsilon_{3}=0.3$ and $\varepsilon_{1} \geqslant 0.02$, Eq. (3) displays rigidly rotating spirals; but if $\varepsilon_{1} \simeq 0.01$ a state containing broken spirals obtains. This breaking of spirals is associated with the onset of spatiotemporal chaos as can be seen qualitatively from local phase portraits (plots in the $e-g$ plane of $\left(e\left(\mathbf{x}, t_{n}\right), g\left(\mathbf{x}, t_{n}\right)\right)$ for fixed spatial coordinate $\mathbf{x}$ and regularly spaced times $t_{n}$ ). Rigidly rotating spirals lead to a periodic temporal evolution; so these portraits show all points collapsing onto a single curve. As spiral breakup occurs these points start to scatter (Fig. 3) indicating the onset of spatiotemporal chaos. Strictly speaking this chaotic behavior is a transient of spatially and temporally irregular behavior whose duration $\tau_{L}$ increases with system size $L$. Fig. 4(a) shows the maximum Lyapunov exponent $\lambda_{m}$ at time $t$ versus $t$ for $L=128$, indicating $\tau_{L=128} \simeq 2200$. This property is in qualitative accord with the experimental finding that the hearts of small mammals are less prone to fibrillation than those of large mammals [9]. For time $t>\tau_{L}$, a quiescent state with $e=g=0$ is obtained. In systems with $L>128, \tau_{L}$ is sufficiently long that a nonequilibrium statistical steady state, displaying spatiotemporal chaos, is established. We find that there are several 

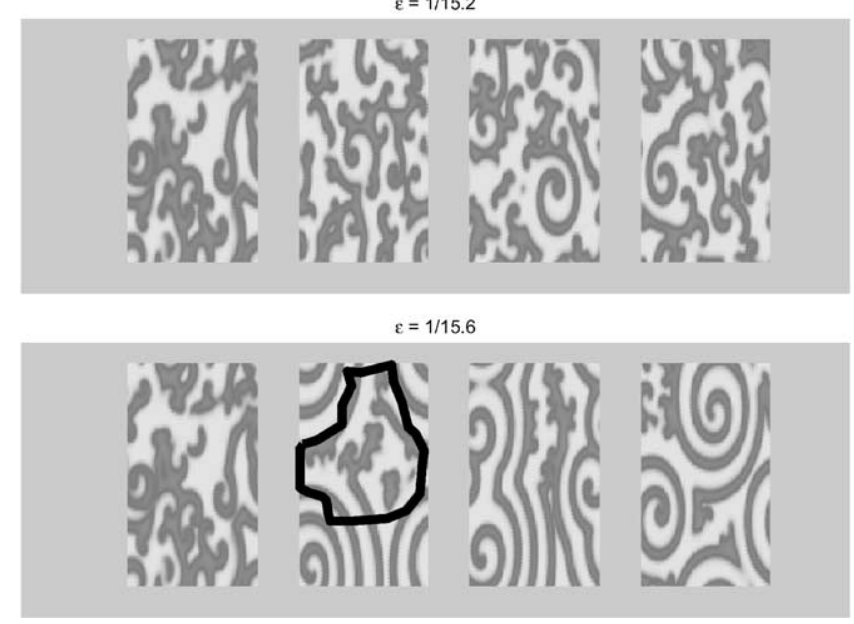

Fig. 2. Pseudocolor plots of the $u$-field for $\epsilon=\frac{1}{15.2} \simeq 0.0657$ (top) and $\epsilon=\frac{1}{15.6} \simeq 0.0641$ (bottom) with configurations (leftmost) prior to a quench from T1 to MP. The second, third and fourth figures are separated by 50000 time units from each other; the first and second figures in each panel are separated by 200000 time units. The black line shows roughly the interface between the state dominated by large spirals and the one dominated by point-like defects.
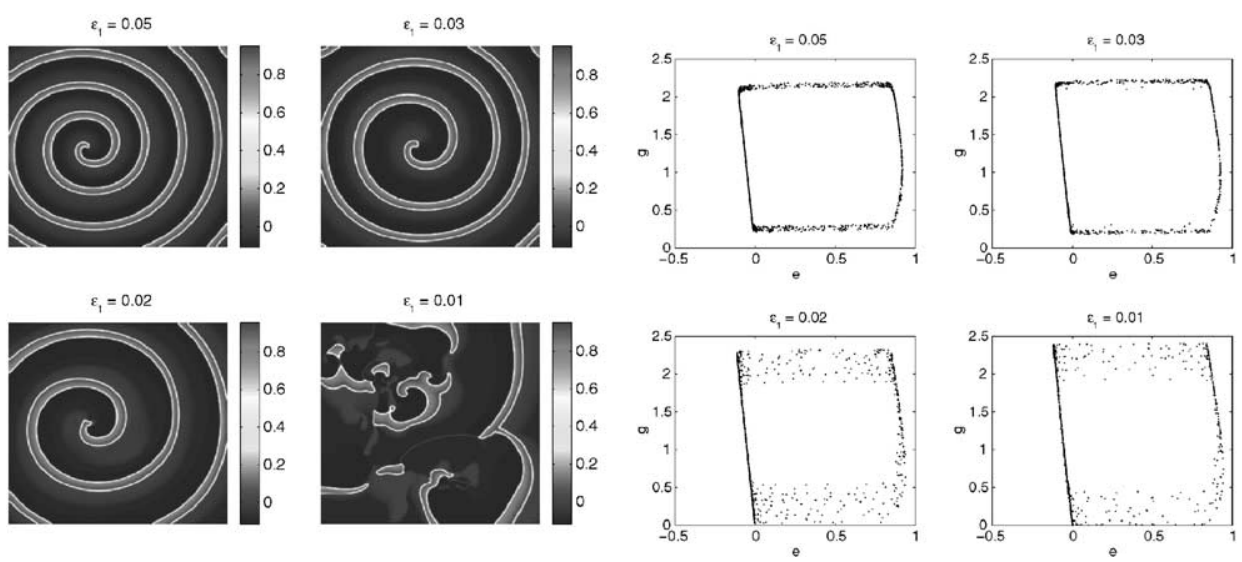

Fig. 3. Pseudocolor plots (left) of the $e$ field (model (3)) at various values of $\varepsilon_{1}$, with other parameter values as given below Eq. (3), and the corresponding local phase portraits (right) reflecting the onset of spiral breakup and spatiotemporal chaos.

positive Lyapunov exponents $\lambda_{i}$ (averages for $\lambda_{i}$ are performed for $\tau_{0}<t<\tau_{L}$, where $\tau_{0}$ is the time of decay for initial transients). The number of positive $\lambda_{i}$ increases with $L$ as does the Kaplan-Yorke dimension $D_{K Y}$ [3,5] [Fig. 4(b)].

It has been suggested [10] that spatiotemporally chaotic states, arising from spiral breakup such as in model (3), are responsible for VF. The control of the resulting 

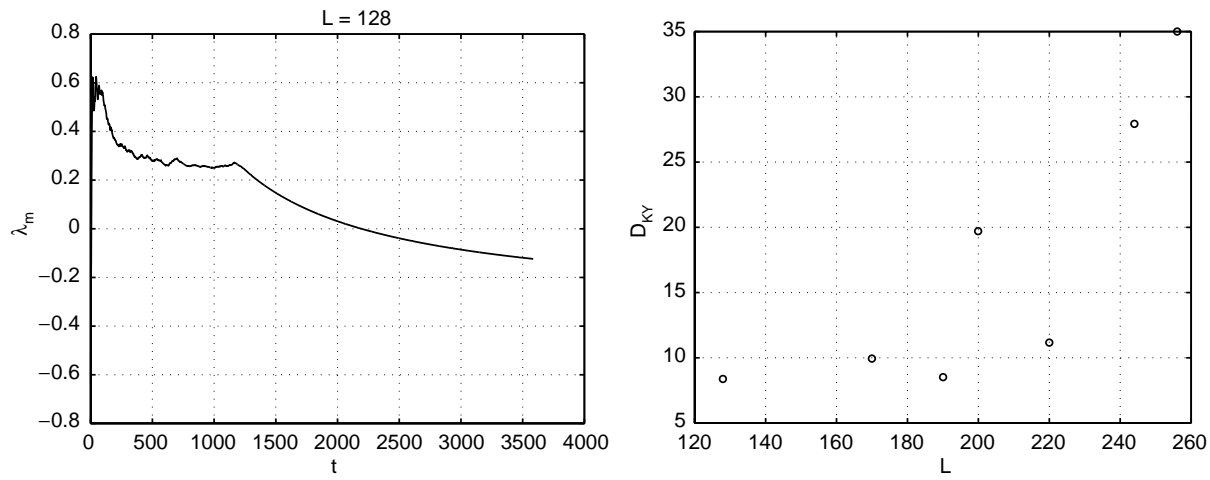

Fig. 4. The maximum Lyapunov exponent $\lambda_{m}$ at time $t$ versus $t$ (left) for Eq. (3) and the parameters given below it. $\lambda_{m}$ approaches a positive constant $(\simeq 0.2)$ and then decays at large times to negative values indicating a long-lived chaotic transient which finally decays to a quiescent state with $e(\vec{x}, t)=0$ and $g(\vec{x}, t)=0$ everywhere. The lifetime of this chaotic transient increases with the size of the system. The Kaplan-Yorke dimension $D_{K Y}$ (right) in the spatiotemporally chaotic transient versus the linear system size $L$ (fluctuations of $D_{K Y}$ for $L \leqslant 220$ arise because of finite-size effects and the varying rate of convergence of $\lambda_{i}$ in our numerical integration with varying $L$ ) [3].

spiral turbulence is being studied actively in models of VF [5,11]. We have proposed an electrical-defibrillation scheme which uses low-amplitude pulses, applied on a coarse grid, to suppress this turbulence. In the model (3), e.g., our scheme drives the system to the spatially and temporally homogeneous state $e=g=0$ rapidly. Then the natural pacemaker of the heart should make it recover from the VF episode.

We divide our $L \times L$ domain into $K^{2}$ smaller blocks by a mesh of line electrodes and choose the block size to be small enough that spirals cannot form [5]. If a pulse is applied to the $e$ field at all points along the mesh for a time $\tau_{c}$, then it effectively simulates Neumann boundary conditions for that time interval for each block in the mesh. $\tau_{c}$ is not large since the individual blocks are of linear size $L / K$ which is so small that it does not sustain long spatiotemporally chaotic transients. Also $K$ need not be very large since the transient lifetime, $\tau_{L}$, decreases rapidly with decreasing $L$. For $d=2, L=512, K=8$ and $\tau_{c}=704 \mathrm{~ms}$ suffices for defibrillation (Fig. 5) if we use a pulse of $250 \mathrm{mV} / \mathrm{ms}$ ( or $250 \mu \mathrm{A} / \mathrm{cm}^{2}$ if $C=1 \mu \mathrm{F} / \mathrm{cm}^{2}$ ). Defibrillation can be obtained with even weaker pulses. We have investigated this systematically [5] for $L=128$, where we show that the minimum pulse amplitude that yields defibrillation is $57.3 \mu \mathrm{A} / \mathrm{cm}^{2}$. This compares favorably with the currents $(\simeq 1 \mathrm{~A})$ delivered by typical defibrillators to the heart muscle.

Our defibrillation method works for a three-dimensional version of model (3) on a $L \times L \times L_{z}$ domain but with the control mesh present only on one $L \times L$ face if $L_{z} \leqslant 4$. A slight modification of this scheme is effective even for $L_{z}>4$ : Instead of applying a pulse for a duration $\tau_{c}$, we apply a sequence of $n$ pulses separated by a time $\tau_{i p}$ and each of duration $\tau_{w}$. We find that, if $L=256$ and $L_{z}=8$, defibrillation occurs in $\simeq 2002 \mathrm{~ms}$ with $\tau_{i p}=22 \mathrm{~ms}, \tau_{w}=0.11 \mathrm{~ms}, n=30$ and a control current density of $57.3 \mu \mathrm{A} / \mathrm{cm}^{2}$. 

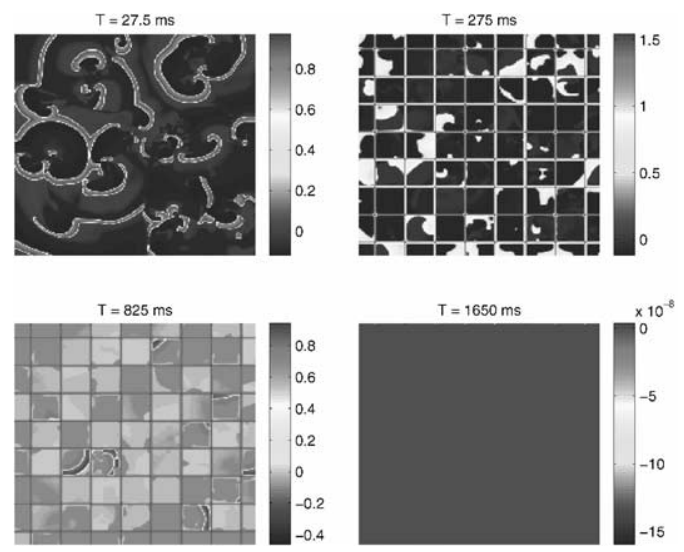

Fig. 5. Pseudocolor plots of the $e$-field illustrating defibrillation by our control scheme in model (3) with $L=512$. The control mesh shows up clearly in the $t=275 \mathrm{~ms}$ panel. A control pulse of amplitude $250 \mathrm{mV} / \mathrm{ms}$ is applied from $t=55$ to $759 \mathrm{~ms}$ to the $e$-field along this mesh. By $t=1650 \mathrm{~ms}$, spatiotemporal chaos is all but eliminated $\left(|e|,|g| \leqslant 10^{-7}\right.$ at all grid points).
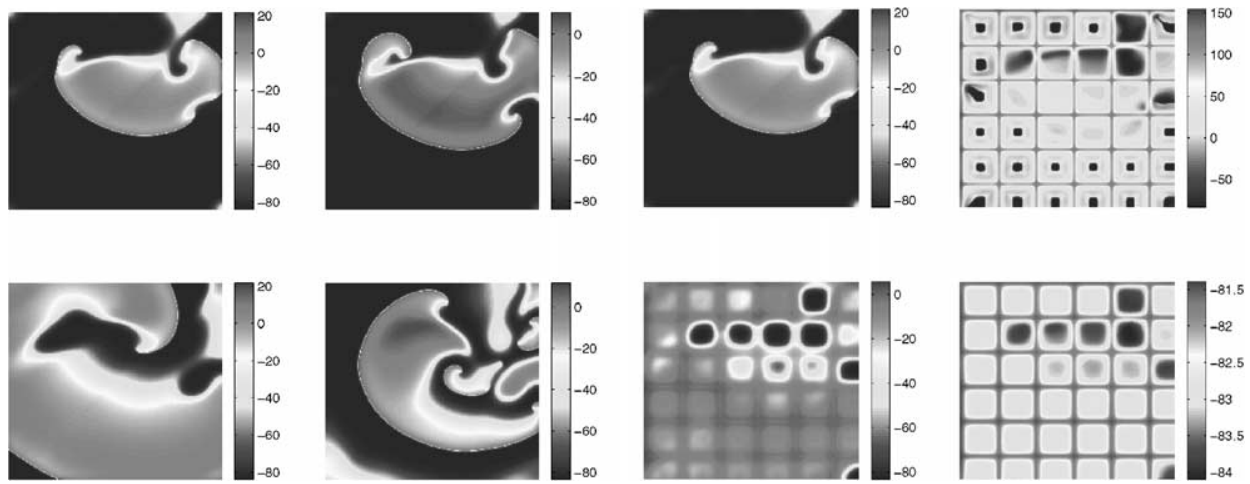

Fig. 6. Pseudocolor plots (left panels) of the membrane potential $V$ in model (2) with $L=200$ for times $t=2700 \mathrm{~ms}$ (top left), $2720 \mathrm{~ms}$ (top right), $2840 \mathrm{~ms}$ (bottom left) and $3020 \mathrm{~ms}$ (bottom right). Pseudocolor plots of $V$ with control (right panels) for the same initial conditions for $t=2700 \mathrm{~ms}$ (top left), $2720 \mathrm{~ms}$ (top right), $2840 \mathrm{~ms}$ (bottom left) and $3020 \mathrm{~ms}$ (bottom right).

We have used our defibrillation scheme to eliminate spiral turbulence in the model (1) and, more important, for the BR model (2). In the BR model we use an $L \times L$ grid with $L=200$ and, by a suitable choice of initial conditions [5], obtain a state with several spirals (top left plot in Fig. 6) at time $t=2500 \mathrm{~ms}$. We then allow this state to evolve both without control (4 plots on the left of Fig. 6) and with control (4 plots on the right of Fig. 6). Our suppression of spiral turbulence is achieved by using a control pulse of $20 \mathrm{mV} / \mathrm{ms}$ (i.e., $20 \mu \mathrm{A} / \mathrm{cm}^{2}$ if we use a capacitance density of $1 \mu \mathrm{F} / \mathrm{cm}^{2}$ ), applied from $t=2701$ to $2820 \mathrm{~ms}$ on the coarse mesh that shows up clearly on the rightmost plots in Fig. 6 . Note that, by $t=3020 \mathrm{~ms}$ defibrillation is more 
or less achieved since the potential $V$ lies in the range -81.5 to $-84 \mathrm{mV}$, which is very close to $-85 \mathrm{mV}$, the resting membrane potential of cardiac tissue and also that of the BR model (2). In model (3) this resting potential is taken to be 0 .

In summary, then, the formal similarity between the model for the oxidation of $\mathrm{CO}$ on $\operatorname{Pt}\left(\begin{array}{lll}1 & 1 & 0\end{array}\right)$ and those for ventricular fibrillation helps us to develop insights about one of these systems by studying the other. We hope that our study will stimulate further studies that exploit this correspondence between these physically different but mathematically similar systems.

\section{Acknowledgements}

We thank CSIR (India) for support and SERC (IISc, Bangalore) for computational facilities.

\section{References}

[1] S. Jakubith, H.H. Rotermund, W. Engel, A. von Oertzen, G. Ertl, Phys. Rev. Lett. 65 (1990) 3013; M. Bär, N. Gottschalk, M. Eiswirth, G. Ertl, J. Chem. Phys. 100 (1994) 1202.

[2] A.T. Winfree (Ed.), Focus issue on fibrillation in normal ventricular myocardium, Chaos 8 (1998) 1.

[3] A. Pande, S. Sinha, R. Pandit, J. Indian Inst. Sci. 79 (1999) 31;

A. Pande, Ph.D. Thesis, Indian Institute of Science, Bangalore, 2001.

[4] A. Pande, R. Pandit, Phys. Rev. E 61 (2000) 6448.

[5] S. Sinha, A. Pande, R. Pandit, Phys. Rev. Lett. 86 (2001) 3678; S. Sinha, A. Sen, A. Pande, R. Pandit, to be published.

[6] M. Hildebrand, M. Bär, M. Eiswirth, Phys. Rev. Lett. 75 (1995) 1503.

[7] G.W. Beeler, H. Reuter, J. Physiol. 268 (1977) 177; M. Courtemanche, Chaos 6 (1996) 579.

[8] A.V. Panfilov, P. Hogeweg, Phys. Lett. A 176 (1993) 295; A.V. Panfilov, Chaos 8 (1998) 57.

[9] Y.-H. Kim, A. Garfinkel, T. Ikeda, T.-J. Wu, C.A. Athill, J.N. Weiss, H.S. Karagueuzian, P.-S. Chen, J. Clin. Invest. 100 (1997) 2486.

[10] R.A. Gray, A.M. Pertsov, J. Jalife, Nature 392 (1998) 75;

F.X. Witkowski, L.J. Leon, P.A. Penkoske, W.R. Giles, M.L. Spano, W.L. Ditto, A.T. Winfree, Nature 392 (1998) 78.

[11] G.V. Osipov, J.J. Collins, Phys. Rev. E 60 (1999) 54;

W.-J. Rappel, F. Fenton, A. Karma, Phys. Rev. Lett. 83 (1999) 456. 